\title{
ROBUST ADAPTIVE POSITION AND FORCE TRACKING CONTROL STRATEGY FOR DOOR-OPENING BEHAVIOUR
}

\author{
Chen, C.-C. ${ }^{* * *} ;$ Li, J.-S., ${ }^{* * * *}$; Luo, J. ; Xie, S.-R. ; Li, H.-Y.*; Pu, H.-Y.* \& Gu, J. \\ ${ }^{*}$ School of Mechatronic Engineering and Automation, Shanghai University, Shanghai, 200072, China \\ ${ }^{* *}$ School of Mechanical and Power Engineering, Henan Polytechnic University, Jiaozuo, 454003, \\ China \\ ${ }^{* * *}$ State Nuclear Power Plant Service Company, Shanghai, 200233, China \\ ${ }^{* * * *}$ Department of Electrical and Computer Engineering, Dalhousie University, Halifax, NS B3H4R2 \\ Canada \\ E-Mail: chenchunchao@hpu.edu.cn, lihengyu@shu.edu.cn
}

\begin{abstract}
The door-opening task is a key step for the indoor rescue and monitoring of a mobile manipulator. However, the contact effect between the gripper and the door handle may produce excessive internal forces to damage mechanical devices because of position errors or the imprecise modelling of the robot and operation environment. To successfully suppress the excessive internal forces and assure the proper posture of the mobile manipulator under holonomic and non-holonomic constraints, a robust adaptive position/force control algorithm was proposed to track the desired posture and force in opening a door to avoid the complexity of compliant mechanism and the unpredictability of the contact stiffness in traditional impedance control. Dynamic simulation studies with MATLAB and RecurDyn were used to verify the dynamic model of the system and obtain the expected positions and forces during door opening. Simulation results and experiments show that the proposed method is robust in modelling errors, joint frictions and environment disturbances and meets the requirement for opening a door with a handle and suppressing excessive internal forces. This study offers reference data and the control method for future real-world door-opening operation in different environments. (Received in August 2015, accepted in March 2016. This paper was with the authors 3 months for 2 revisions.)
\end{abstract}

Key Words: Tracking Simulation, Door Opening, Manipulator, Robust Adaptive Control

\section{INTRODUCTION}

Mobile manipulators are increasingly used in the rescue service field and usually require performing many door-opening and -closing tasks. The autonomous door-opening control of mobile manipulators is a challenging research topic in the automatic control field. Autonomous door-opening control studies extend the working space of mobile manipulators and significantly improve the robot service ability for human beings.

Performing door-opening task with mobile manipulators usually requires sophisticated control or mechanical designs. Large internal forces can be generated because of the inevitable measurement errors of sensors, the positioning errors of the mobile base and the different types of interferences. Excessive internal forces may cause the task to fail or cause damage to the handle and the gripper. Therefore, studying the hybrid position/force control of the mobile manipulator and the coordination control between the manipulator and the mobile platform is highly significant in theoretical studies and practical application.

\section{STATE OF THE ART}

Considerable existing literature has focused on compliant mechanisms or active compliant control based on sensor feedback to solve the problem of excessive internal forces. Special compliant mechanisms have been explored to adjust contact stiffness and to reduce large internal forces [1]. Liu et al. [2] developed a mobile modular reconfigurable robot to solve the 
door-opening problem by reasonably selecting the working modes of the joint module with adaptive compliance control and minimal computational complexity. Kessens et al. [3] proposed a simple, low-cost method to resolve forces non-tangent to the motion path, and the joint compliance was achieved by a clutch mechanism located in the manipulator joint motors.

Many researchers have devoted themselves to examining the feedback control of multiple sensors. Meeussen et al. [4] developed an autonomous robotic system to open doors and plug itself into electrical outlets to recharge by integrating compliant control into manipulation and by combining vision and laser sensor motions. Prats et al. [5] proposed a new vision-tactileforce approach to combine tactile information with vision and force feedback to open a sliding door. Winiarski et al. [6] considered force and velocity constraints in the control law to avoid environment damage in opening a door, and the impedance control law was expanded to achieve the safe behaviour of the direct position/force controller of the KUKA LWR4+ robot. Zucker et al. [7] emphasised the generating of smooth trajectories that minimised the unnecessary door-opening motion of a humanoid robot to avoid excessive forces. Guo et al. [8] applied the hybrid position/force control in opening doors to generate the velocity of the end-effector, and the coordinated motion control of the mobile manipulator was adopted to drive the entire system to a suitable position to open doors successfully. Kim et al. [9] proposed a twisting door handle strategy, in which the compliant motion control was used to overcome the limitation of uncertain information from the manipulator and to estimate the centre of the rotation point of the door handle. Chung et al. [10] applied active sensing algorithms to a multifingered robotic hand in a service robot PSR1 to diminish uncertainties in the environment. Fingertip tactile sensors provided information on the contact position and the contact force for compliance control during the door-opening process. Kim et al. [11] adopted the double-active universal joint with inexpensive force sensors in the manipulator joint mechanism of a home service robot for the door-opening task. Lee et al. [12] offered a novel stiffness modulation method for impedance control, which incorporated real-time optimisation for approaching, grasping, rotating and pulling the door handle under a semiautonomous operation. A wrist-mounted force sensor could provide supplementary information to estimate some 3D features. Karayiannidis et al. [13] proposed a door-opening method without requiring prior knowledge of the door kinematics. A force controller regulated the radial force to a desired small value, and the velocity controller ensured that the end effector of the robot moved with the desired tangential velocity. Schmid et al. [14] offered a multisensor-based generic approach to open doors. A visual algorithm was used to localise the handle, and the door-opening task was accomplished with the information fusion of a force-torque sensor in the robot wrist and a tactile sensor matrix in the robot gripper. Winiarski and Banachowicz [15] proposed an impedance control approach to open a cabinet door with a seven-degree-of-freedom (DOF) redundant manipulator.

These researchers were successful in their studies. However, the compliant mechanisms and force sensors can change the dynamic characteristics of the manipulator. Compliant mechanisms are complex in structure, and the noises of force sensors can cause system instability. Inappropriate contact stiffness values in the traditional impedance control may cause the failure of the door-opening task. The hybrid position/force methods in existing literature rarely involve the robustness and non-holonomic constraints of the system.

Therefore, this study proposes a new door-opening control approach based on the robust adaptive position/force tracking control to adapt to a narrow operating environment. The proposed controller has the ability to overcome dynamic uncertainties, friction torques, variable payloads, external torque disturbances and measurement noises, which are usually present in practical environments [16-17]. The robust adaptive control technique can 
effectively compensate for system uncertainties and simultaneously suppress external disturbances [18-20].

The remainder of this paper is organised as follows. Section 3 defines the reference paths of the manipulator in the operating space and describes the robust adaptive position/force tracking control strategy. Section 4 presents the simulation results based on the proposed control method. Section 5 concludes the study.

\section{METHODOLOGY}

\subsection{Kinematics for door opening}

This study focuses on the door-opening method of a mobile manipulator. The following assumptions can be made. 1) The actual position of the vehicle and the door can be measured by a motor encoder and vision. 2) The rotation axis of the door is always perpendicular to the floor, which guarantees the door handle to move on the horizontal plane. 3) The current dooropening angle starts from $0^{\circ}$, and the rotation axis of the wrist joint is perpendicular to the rotation axis of the door, thus guaranteeing the direction of the tangent force. 4) The door parameters are calculated according to sensor datum.

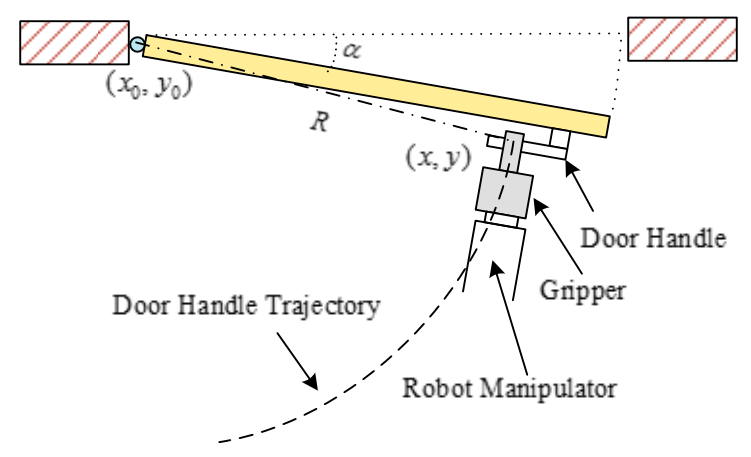

Figure 1: Schematic diagram of door opening.

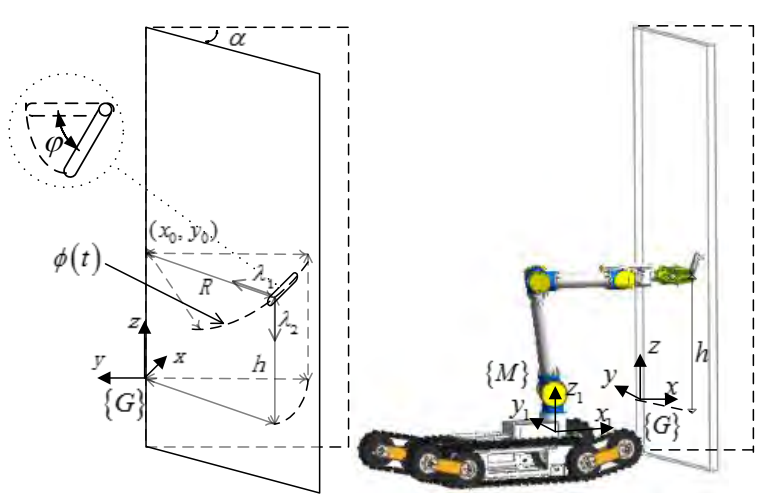

Figure 2: Kinematic parameters for door opening.

When opening a door, the vehicle remains still or moves backward and forward to reduce power consumption and make the robot suitable for a narrow environment. The robot endeffector tracks the desired trajectory to approach and rotate the handle for unbolting the door. When pulling the handle, the robot end-effector is constrained to move along the circular trajectory of door handle, and the occurrence of large internal forces is inevitable because of the tracking and modelling errors. The schematic of door opening is illustrated in Fig. 1.

The kinematic parameters of door opening are depicted in Fig. 2. The global coordinate frame $\{G\}$ and the local coordinate frame $\{M\}$ are fixed on the door hinge and on the connection point of the manipulator and the vehicle, respectively. Relative to reference frame $\{G\}$, the gripper trajectory has a centre of a circle $\left(x_{0}, y_{0}\right)$ and the radius vector $R$. The angular velocity vector $\dot{\alpha}$ and the instantaneous velocity vector $\dot{p}_{e}$ along the motion direction satisfy the relation $\dot{p}_{e}=R \times \dot{\alpha}$ when $p_{e}$ is the current position vector of the gripper, and $\alpha$ is the rotation angle vector. Physical truth presents that the radial motion velocity is zero (i.e. $R^{T} \dot{p}_{e}=0$ ). The radial component force $F_{R}$ of the contact force $F$ in the gripper when pulling the door handle is small and is expressed as $F_{R}=R^{T} F$.

\subsection{Motion planning of the robot manipulator}

The proposed door-opening method is summarised as follows [21]: 
1) The fixed position of the vehicle and the reference trajectories of the manipulator are computed on the basis of the door parameters and the operation space of the manipulator.

2) The vehicle moves to the desired position, and the gripper then moves close to the door handle along the designed trajectory.

3) The door handle is grasped and turned to unbolt the door.

4) The handle is gripped, the door is pulled open slowly, and the motion trajectory is adjusted by the adaptive position/force controller to restrain the amplitude of contact force.

To complete the above operations, the optimized path planning should be considered first [22]. Only the path planning of the manipulator is considered in this study given that the vehicle remains still during the door-opening process. The planned trajectories (Fig. 3) include three segments: approaching the handle, rotating the handle and pulling the door open. Hermite cubic spline functions are used in the planned trajectories to assure smooth movement. The trajectories in approaching and rotating the handle are planned to avoid collision with the door or the handle. The elastic resistance and friction resistance of the handle joint, which are approximately proportional to the rotation angle of the handle, are exerted on the end-effector when the gripper rotates the handle. The resistance may be considered a time-varying payload on the end-effector of the manipulator. The planned trajectory when pulling the door open is restricted to the handle path because of kinematic complaints. The mechanical constraints (i.e. joint limits, maximum speeds and maximum applicable torque at each joint module) in the door-opening process are considered at all times.

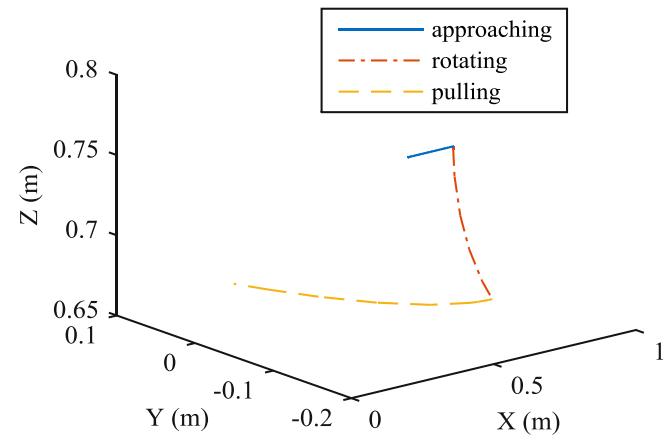

Figure 3: Planned trajectories of the end-effector.

As the wrist joint axis is perpendicular to the rotational axis of the door when opening the door, the ZYZ Euler angles of the end-effector satisfy the relations $\alpha=q_{1}, \beta=q_{2}+q_{3}+q_{4}$ and $\gamma=q_{5}$, where $q_{i}(i=1, \ldots, 5)$ denotes the $i^{\text {th }}$ joint angle of the manipulator, and $\gamma$ changes with the rotation angle of the handle to align the gripper with the handle.

\subsection{Dynamics of the mobile manipulator}

The dynamics of the mobile manipulator can be expressed as:

$$
M(q) \ddot{q}+C(q, \dot{q}) \dot{q}+G(q)+d(t)=H(q) \tau+J^{T} \lambda
$$

where $q, \dot{q}$ and $\ddot{q}$ represent the generalised coordinates, velocity and acceleration, respectively; $M(q) \in R^{n \times n}$ is the inertia matrix; $C(q, \dot{q}) \in R^{n \times n}$ is the matrix that contains the Coriolis and centrifugal forces; $G(q) \in R^{n}$ is the gravity; $d(t) \in R^{n}$ is the disturbance moment at the joints; $\lambda \in R^{m}$ is the generalised force multiplier; $J \in R^{m \times n}$ is the constraint matrix; $H(q) \in R^{n \times m}$ is the full-rank input transformation matrix; and $\tau \in R^{m}$ is the input torque vector.

The generalised coordinates of the mobile manipulator are denoted as $q=\left[q_{b}, q_{a}\right]^{T}$, in which $q_{b} \in R^{n_{b}}$ and $q_{a} \in R^{n_{a}}$ represent the generalised coordinates of the vehicle and the 
manipulator, respectively. $M(q)=\left[\begin{array}{cc}M_{b} & M_{b a} \\ M_{a b} & M_{a}\end{array}\right], C(q, \dot{q})=\left[\begin{array}{cc}C_{b} & C_{b a} \\ C_{a b} & C_{a}\end{array}\right], H(q)=\left[\begin{array}{cc}H_{b} & 0 \\ 0 & H_{a}\end{array}\right]$, $J=\left[\begin{array}{cc}A & 0 \\ J_{b} & J_{a}\end{array}\right], G(q)=\left[\begin{array}{l}G_{b} \\ G_{a}\end{array}\right]$ and $d(t)=\left[\begin{array}{l}d_{b} \\ d_{a}\end{array}\right]$ denote the expanded dynamic parameters. The constraint Lagrangian multipliers can be expressed as $\lambda=\left[\lambda_{b}, \lambda_{a}\right]^{T}$, where $\lambda_{b}$ represents the non-holonomic constraint, and $\lambda_{\mathrm{a}}$ is the holonomic constraint. Supposing that the vehicle is subjected to $l$ non-holonomic constraints, the constraint relation can be expressed as:

$$
A\left(q_{b}\right) \dot{q}_{b}=0
$$

where $A\left(q_{b}\right) \in R^{l \times n_{b}}$ is the kinematic constraint matrix with full rank $l$.

An $n_{b}-l$ rank matrix $N\left(q_{b}\right) \in R^{n_{b} \times\left(n_{b}-l\right)}$ may exist in the null space of $A\left(q_{b}\right)$ and satisfy $N^{T}\left(q_{b}\right) A^{T}\left(q_{b}\right)=0$. This matrix implies the existence of vector $\dot{\eta}$ and satisfies the relation $\dot{q}_{b}=N\left(q_{b}\right) \dot{\eta}$. The dynamics of the mobile manipulator can be rewritten as:

$$
M_{\zeta} \ddot{\zeta}+C_{\zeta} \dot{\zeta}+G_{\zeta}+d_{\zeta}=H_{\zeta} \tau+J_{\zeta}{ }^{T} \lambda_{\zeta}
$$

where $M_{\zeta}=\left[\begin{array}{cc}N^{T} M_{b} N & N^{T} M_{b a} \\ M_{a b} N & M_{a}\end{array}\right], C_{\zeta}=\left[\begin{array}{cc}N^{T} M_{b} \dot{N}+N^{T} C_{b} N & N^{T} C_{b a} \\ M_{a b} \dot{N}+C_{a b} N & C_{a}\end{array}\right], G_{\zeta}=\left[\begin{array}{c}N^{T} G_{b} \\ G_{a}\end{array}\right]$, $d_{\zeta}=\left[\begin{array}{c}N^{T} d_{b} \\ d_{a}\end{array}\right], H_{\zeta}=\left[\begin{array}{cc}N^{T} H_{b} & 0 \\ 0 & H_{a}\end{array}\right], \lambda_{\zeta}=\left[\begin{array}{c}0 \\ \lambda_{a}\end{array}\right], J_{\zeta}=\left[\begin{array}{cc}0 & 0 \\ J_{b} & J_{a}\end{array}\right]$ and $\zeta=\left\lfloor\begin{array}{l}\eta \\ q_{a}\end{array}\right\rfloor$.

When the system is subjected to $k$ independent holonomic constraints, the constraint function can be expressed as $\phi(\zeta)=0$ with $\zeta=\left[\begin{array}{ll}\eta & q_{a}\end{array}\right]^{T}$, and $\phi(\zeta) \in R^{k}$ is twice the continuous differentiable with a constraint Jacobian matrix $J_{\zeta}=\partial \phi / \partial \zeta$ and the relation $J_{\zeta}(\zeta) \dot{\zeta}=0$. Vector $q_{a}$ can be divided into constrained variables and independent variables: $q_{a}=\left[\begin{array}{ll}q_{a}{ }^{1} & q_{a}{ }^{2}\end{array}\right]^{T}$. Thus, $\zeta=\left[\begin{array}{lll}\eta & q_{a}{ }^{1} & q_{a}{ }^{2}\end{array}\right]^{T}$. Accumulated knowledge agrees that the function $\zeta=\psi(\xi)$ with $\xi=\left[\begin{array}{ll}\eta & q_{a}^{1}\end{array}\right]^{T}$ exists, and $\dot{\zeta}=L(\xi) \dot{\xi}$ with $L(\xi)=\partial \psi(\xi) / \partial \xi$ can be obtained. $J^{1 T}(\xi)=J_{\zeta}{ }^{T}(\psi(\xi))$ and $L^{T}(\xi) J^{1 T}(\xi)=0$ can be derived from the above descriptions. A reduced order dynamic equation can be obtained as follows:

$$
M^{1} L(\xi) \ddot{\xi}+C^{1} \dot{\xi}+G^{1}+d^{1}(t)=u+J^{1 T} \lambda_{a}
$$

where $M^{1}=\left[\begin{array}{cc}N^{T} M_{b} N & N^{T} M_{b a} \\ M_{a b} N & M_{a}\end{array}\right], C^{1}=\left[\begin{array}{cc}N^{T} M_{b} N & N^{T} M_{b a} \\ M_{a b} N & M_{a}\end{array}\right] \dot{L}+\left[\begin{array}{cc}N^{T} M_{b} \dot{N}+N^{T} C_{b} N & N^{T} C_{b a} \\ M_{a b} \dot{N}+C_{a b} N & C_{a}\end{array}\right] L$, $G^{1}=\left[\begin{array}{c}N^{T} G_{b} \\ G_{a}\end{array}\right], d^{1}(t)=\left[\begin{array}{c}N^{T} d_{b} \\ d_{a}\end{array}\right], H^{1}=\left[\begin{array}{cc}N^{T} H_{b} & 0 \\ 0 & H_{a}\end{array}\right]$ and $u=\left[\begin{array}{cc}N^{T} H_{b} & 0 \\ 0 & H_{a}\end{array}\right]\left[\begin{array}{l}\tau_{b} \\ \tau_{a}\end{array}\right]$.

The following expression can be obtained by multiplying $L^{T}$ on both sides of Eq. (4):

$$
M_{L} \ddot{\xi}+C_{L} \dot{\xi}+G_{L}+d_{L}(t)=L^{T} u
$$

where $M_{L}=L^{T} M^{1} L, C_{L}=L^{T} C^{1}, G_{L}=L^{T} G^{1}$ and $d_{L}(t)=L^{T} d^{1}(t)$.

The controller can be divided into position controller $u_{p}$ and force controller $u_{f}$ :

$$
u=u_{p}-J^{1 T} u_{f}
$$

The following expression can be obtained according to Eqs. (4) to (6):

$$
M_{L} \ddot{\xi}+C_{L} \dot{\xi}+G_{L}+d_{L}(t)=L^{T} u_{p}
$$




$$
\lambda_{a}=Z\left(C^{1} \dot{\xi}+G^{1}+d^{1}(t)-u_{p}\right)+u_{f}
$$

where $Z=\left(J^{1}\left(M^{1}\right)^{-1} J^{1 T}\right)^{-1} J^{1}\left(M^{1}\right)^{-1}$ is the weighted inverse matrix of $J$, and $L^{+}=\left(L^{T} L\right)^{-1} L^{T}$ is the pseudo inverse matrix of $L$.

System errors for the desired trajectory $\xi_{d}$ and the desired force $\lambda_{a d}$ can be defined as $e=\xi-\xi_{d}, \dot{\xi}_{r}=\dot{\xi}_{d}-\Lambda e, s=\dot{e}+\Lambda e$ and $e_{\lambda}=\lambda_{a}-\lambda_{a d}$, where $\Lambda$ is a positive constant. $\xi, \dot{\xi}$, $\xi_{r}, \dot{\xi}_{r}, M_{L}, C_{L}, G_{L}$ and $d_{L}$ are bounded for the real system.

\subsection{Model-based control}

Without considering parameter uncertainty and external disturbances, the control laws based on the model are designed as follows:

$$
\begin{gathered}
L^{T} u_{p}=-K_{p} s+\Phi_{L} \\
u_{f}=Z L^{+T} M_{L} \ddot{\xi}_{d}+\lambda_{a d}-K_{\lambda} e_{\lambda}-K_{I} \int_{0}^{t} e_{\lambda} d \tau
\end{gathered}
$$

where $\Phi_{L}=\Psi_{L} \Theta_{L} ; \Psi_{L}=\left[M_{L}, C_{L}, G_{L}\right] ; \Theta_{L}=\left[\ddot{\xi}_{r}, \dot{\xi}_{r}, 1\right]^{T} ; K_{p}, K_{\lambda}$ and $K_{I}$ are the symmetric gain matrices.

Proof $(i)$ : The closed-loop system dynamics can be given as:

$$
M_{L} \dot{s}=-K_{p} s-C_{L} s
$$

The Lyapunov function candidate is expressed as:

$$
V=\frac{1}{2} s^{T} M_{L} S
$$

The time derivative of $V$ is:

$$
\begin{aligned}
\dot{V} & =s^{T} M_{L} \dot{s}+\frac{1}{2} s^{T} \dot{M}_{L} s \\
& =s^{T}\left(-K_{p} s-C_{L} s\right)+\frac{1}{2} s^{T} \dot{M}_{L} s \\
& =-s^{T} K_{p} s \leq-\lambda_{\min }\left(K_{p}\right)\|s\|^{2} \leq 0
\end{aligned}
$$

The integration of the above equation can be expressed as $V(t)-V(0) \leq-\int_{0}^{t} s^{T} K_{p} s d \tau \leq 0$, which implies that $s \in L_{\infty}^{n-k-l}$ and $s \in L_{2}^{n-k-l}$. From $s=\dot{e}+\Lambda e, e, \dot{e} \in L_{\infty}$. According to $\xi, \dot{\xi}, \xi_{r}, \dot{\xi}_{r} \in L_{\infty}^{n-k-l}$ and $e, \dot{e} \in L_{\infty}$, we can conclude that $\dot{s}$ and $\ddot{\xi}$ are bounded. $s \rightarrow 0$ as $t \rightarrow \infty$.

Proof (ii): Substituting Eqs. (9) and (10) into Eq. (8) yields

$$
\left(I+K_{\lambda}\right) e_{\lambda}+K_{I} \int_{0}^{t} e_{\lambda} d \tau=-Z L^{+T} M_{L} \ddot{\xi}+Z L^{+T} M_{L} \ddot{\xi}_{d}
$$

The right side of Eq. (13) and $e_{\lambda}$ are bounded given that $\ddot{\xi}, \ddot{\xi}_{d}$ and $Z$ are bounded, and $\xi \rightarrow \xi_{d}$. Thus, $\tau$ is bounded.

\subsection{Robust adaptive control}

The hybrid position/force controller is applied to adjust the excessive contact force induced by modelling uncertainty and measurement errors to ensure the tracking performance when the gripper follows the reference trajectory to pull the door handle.

The following assumptions are established to facilitate robust adaptive control formulation: 
1) The joint references $\xi$ and $\xi_{d}$ are bounded and have second-order-bounded uniformly continuous derivatives.

2) The friction model $f_{r}=b \dot{\xi}+f_{c} \operatorname{sgn}(\dot{\xi})$ and other disturbances $\Delta d$ are integrated into item $d_{L}$. Some finite positive constants $\rho_{i}>0(1 \leq i \leq 2)$ and finite nonnegative constant $\rho_{3} \geq 0$ exist. The damping coefficient $b$, friction coefficient $f_{c}$ and other disturbances $\Delta d$ are all bounded and satisfy the inequalities $\|b\| \leq \rho_{1},\left\|f_{c}\right\| \leq \rho_{2}$ and $\|\Delta d\| \leq \rho_{3}$. Therefore, the disturbance item $d_{L}=Y_{r} a_{r}$, where $Y_{r}=[\|\dot{\xi}\|,\|\operatorname{sgn}(\dot{\xi})\|, 1]$ and $a_{r}=\left[\rho_{1}, \rho_{2}, \rho_{3}\right]^{T}$.

Given system uncertainties, joint frictions and other interferences with the nominal parameters $\hat{M}_{L}, \hat{C}_{L}$ and $\hat{G}_{L}$, the dynamic Eq. (7) can be rewritten as:

$$
\hat{M}_{L} \ddot{\xi}+\hat{C}_{L} \dot{\xi}+\hat{G}_{L}-\tilde{\Psi} \Theta+Y_{r} a_{r}=L^{T} u_{p}
$$

where $\tilde{\Psi}=\left[\hat{M}_{L}-M_{L}, \hat{C}_{L}-C_{L}, \hat{G}_{L}-G_{L}\right]$ and $\Theta=[\ddot{\xi}, \dot{\xi}, 1]^{T}$.

The control laws are considered as follows:

$$
\begin{gathered}
L^{T} u_{p}=-K_{p} s+\left(\hat{M}_{L} \ddot{\xi}_{r}+\hat{C}_{L} \dot{\xi}_{r}+\hat{G}_{L}\right)+u_{m} \Theta-\frac{s\left(Y_{r} \hat{a}_{r}\right)^{2}}{\|s\| Y_{r} \hat{a}_{r}+\delta} \\
u_{f}=\frac{\hat{\chi}^{2}}{\hat{\chi}+\delta} \ddot{\xi}_{d}+\lambda_{d}-K_{\lambda} e_{\lambda}-K_{I} \int_{0}^{t} e_{\lambda} d \tau \\
u_{m}=-k \int_{0}^{t} \Theta^{T} s d \tau \\
\dot{\hat{a}}_{r}=-\Gamma Y_{r}^{T} s \\
\hat{\chi}=\left\|\hat{Z} L^{+T} \hat{M}_{L}\right\|
\end{gathered}
$$

where $\hat{Z}=\left(J^{1} \hat{M}^{-1} J^{1 T}\right)^{-1} J^{1 T} \hat{M}^{-1} ; K_{p}$ and $\Gamma$ are the symmetric positive definite gain matrices; $\delta$ and $k$ are the positive constants; and $\hat{a}_{r}=\left[\begin{array}{lll}\hat{\rho}_{1} & \hat{\rho}_{2} & \hat{\rho}_{3}\end{array}\right]$ is the estimation of $a_{r}$.

The following stability analysis is made given the convergence of the controller.

Proof $(i)$ : The closed-loop dynamics is obtained as follows:

$$
\hat{M}_{L} \dot{s}=-K_{p} s-\hat{C}_{L} s+\tilde{\Psi} \Theta-Y_{r} a_{r}+u_{m} \Theta-\frac{s\left(Y_{r} \hat{a}_{r}\right)^{2}}{\|s\| Y_{r} \hat{a}_{r}+\delta}
$$

The Lyapunov function candidate is given as,

$$
V=\frac{1}{2} s^{T} \hat{M}_{L} s+\frac{1}{2} k \varepsilon^{T} \varepsilon+\frac{1}{2} \tilde{a}_{r}^{T} \Gamma^{-1} \tilde{a}_{r}
$$

where $\varepsilon=\frac{1}{k} \tilde{\Psi}-\int_{0}^{t} \Theta^{T} s d \tau$ and $\tilde{a}_{r}=a_{r}-\hat{a}_{r} . u_{m} \rightarrow-\tilde{\Psi}$, and $\dot{\varepsilon}=-s \Theta$ when $\varepsilon \rightarrow 0$.

The time derivative of $V$ can be obtained as:

$$
\begin{aligned}
\dot{V} & =s^{T} \hat{M}_{L} \dot{s}+\frac{1}{2} s^{T} \dot{\hat{M}}_{L} s+k \varepsilon^{T} \dot{\varepsilon}+\tilde{a}_{r}^{T} \Gamma^{-1} \dot{\tilde{a}}_{r} \\
& =-s^{T} K_{p} s+s^{T}\left(\tilde{\Psi} \Theta+u_{m} \Theta-Y_{r} a_{r}-\frac{s^{T} s\left(Y_{r} \hat{a}_{r}\right)^{2}}{\|s\| Y_{r} \hat{a}_{r}+\delta}\right)-k \varepsilon^{T} s \Theta+\tilde{a}_{r}^{T} Y_{r}^{T} s \\
& =-s^{T} K_{p} s-s^{T} Y_{r} a_{r}-\frac{s^{T} s\left(Y_{r} \hat{a}_{r}\right)^{2}}{\|s\| Y_{r} \hat{a}_{r}+\delta}+s^{T} Y_{r} \tilde{a}_{r} \\
& \leq-s^{T} K_{p} s+\delta \leq-\lambda_{\min }\left(K_{p}\right)\|s\|^{2}+\delta
\end{aligned}
$$


When $\|s\| \geq \sqrt{\delta_{\max } / \lambda_{\min }\left(K_{p}\right)}, \dot{V} \leq 0$, and $V(t)-V(0) \leq 0$. The above proof indicates that $s \in L_{\infty}$ and $s \in L_{2}$. Thus, $e, \dot{e} \in L_{\infty}$. The above assumptions prove that the signals $\xi, \dot{\xi}, \ddot{\xi}, \xi_{r}$, $\dot{\xi}_{r}, \ddot{\xi}_{r}, \Theta$ and $\Psi$ are all bounded. The closed-loop dynamics Eq. (20) shows that $\dot{s} \in L_{\infty}$ and $\ddot{\xi} \in L_{\infty}$. Finally, Barbalat's lemma can be applied to conclude that $s \rightarrow 0$ as $t \rightarrow \infty$. Thus, $e \rightarrow 0$ and $\dot{e} \rightarrow 0$ as $t \rightarrow \infty$.

Proof (ii): The force error closed-loop equation can be obtained by substituting Eqs. (15) and (16) into Eq. (8):

$$
\left(I+K_{\lambda}\right) e_{\lambda}+K_{I} \int_{0}^{t} e_{\lambda} d \tau=-Z L^{+T} M_{L} \ddot{\xi}+\frac{\hat{\chi}^{2}}{\hat{\chi}+\delta} \ddot{\xi}_{d}
$$

$\dot{\xi}$ and $\hat{Z}$ are bounded, and $\xi \rightarrow \xi_{d}$. Thus, $-Z L^{+T} M_{L} \ddot{\xi}+\frac{\hat{\chi}^{2}}{\hat{\chi}+\delta} \ddot{\xi}_{d}$ is bounded. Therefore, $e_{\lambda}$ is bounded, and $e_{\lambda} \rightarrow 0$ as $t \rightarrow \infty$.

\section{SIMULATION RESULT ANALYSIS}

Two simulation results are presented in this section to verify the proposed method. The first one is a numerical example, in which the emphasis is on the theoretical analysis of the dynamics about the mobile manipulator. This example is used to illustrate the case in which the proposed control method is used to track the predefined trajectories and contact forces. This method is then applied to a virtual door-opening environment to prove the effectiveness of the robust adaptive controller. The simulation of this virtual model meets actual problems because of the designed parameters from the real door-opening scenario.

\subsection{Numerical example}

The proposed door-opening method is validated through a series of computer simulations in MATLAB/Simulink, and all constraints are considered throughout the door-opening process. This study selected the desired trajectories in the working space of the manipulator and in the safe margin of the manipulator without collision with the door. The simulations show that all joints move within their mechanical limits.

The link lengths of the manipulator in this study are $L_{1}=0.245 \mathrm{~m}, L_{2}=0.500 \mathrm{~m}$, $L_{3}=0.400 \mathrm{~m}$, and $L_{4}=0.317 \mathrm{~m}$. The link offset is $d=0.092 \mathrm{~m}$, and the qualities of the links are $m_{1}=4.56 \mathrm{~kg}, m_{2}=8.77 \mathrm{~kg}, m_{3}=5.00 \mathrm{~kg}$, and $m_{4}=4.29 \mathrm{~kg}$. Each joint angle of the manipulator is as follows: $q_{1}, q_{3}, q_{4} \in\left[-150^{\circ}, 150^{\circ}\right], q_{2} \in\left[-110^{\circ}, 110^{\circ}\right]$ and $q_{5} \in\left[-180^{\circ}, 180^{\circ}\right]$.

The functions of the reference trajectories about time parameter $t$ are defined as

$$
\text { a) }\left[\begin{array}{l}
x \\
y \\
z
\end{array}\right]=\left[\begin{array}{c}
0.5+0.04 t \\
-0.09 \\
0.76
\end{array}\right] \text {, b) }\left[\begin{array}{l}
x \\
y \\
z
\end{array}\right]=\left[\begin{array}{c}
0.66 \\
-0.19+0.1 \cos (\theta) \\
0.76+0.1 \sin (\theta)
\end{array}\right] \text { and c) }\left[\begin{array}{l}
x \\
y \\
z
\end{array}\right]=\left[\begin{array}{c}
0.66+0.69 \cos (\theta) \\
-0.55+0.69 \sin (\theta) \\
0.6734
\end{array}\right] \text {, }
$$

where a) describes a line trajectory for approaching the handle, and b) and c) represent the two arc trajectories of rotating and pulling the handle with the parameters, $\theta \in\left[-60^{\circ}, 0\right]$ and $\theta \in\left[-135^{\circ},-90^{\circ}\right]$, respectively.

The Euler angles $\beta$ and $\gamma$ are defined as $\beta=\pi / 2$ and $\gamma=\pi / 2-\pi \cdot \operatorname{satlin}(0.5 t-4.5) / 2$, respectively, where $\operatorname{satlin}(\cdot)$ is a saturated linear function, and $t$ is a time parameter. The friction and the disturbance exerted on the joints are assumed as $f_{r}=\dot{q}+0.5 \operatorname{sgn}(\dot{q})$ and 
$\tau_{d i s}=[\sin (3 t), \sin (3 t), \sin (3 t), \sin (3 t), \sin (3 t)]^{T}$. The reference forces on the gripper are set to $\lambda_{a d}=\left[\begin{array}{llll}0.5 \mathrm{~N}, & 0.5 \mathrm{~N}\end{array}\right]^{T}$. The manipulator is subjected to two constraints, namely, $\phi_{1}=\left(x_{e}(t)-x_{0}\right)^{2}+\left(y_{e}(t)-y_{0}\right)^{2}-R^{2}$ and $\phi_{2}=z_{e}(t)-h_{0}$, where $x_{0}=0.718 \mathrm{~m}, y_{0}=0.556 \mathrm{~m}$ and $h_{0}=0.745 \mathrm{~m}$. The above mentioned definitions are the functions about time parameter $t$.
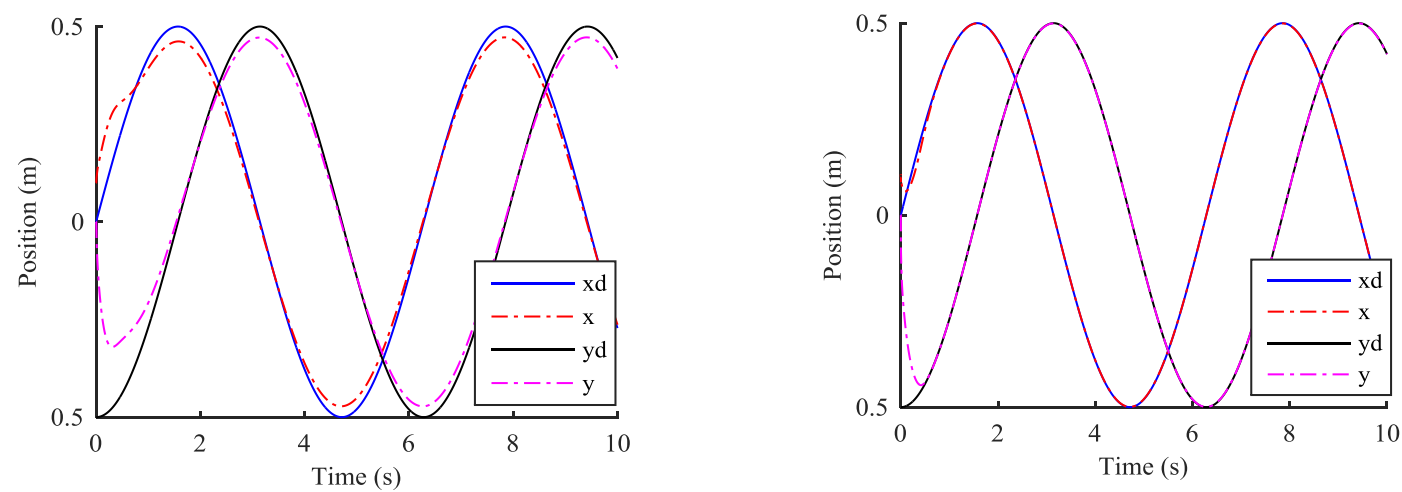

Figure 4: Trajectory tracking of the vehicle under model-based control and robust adaptive control.
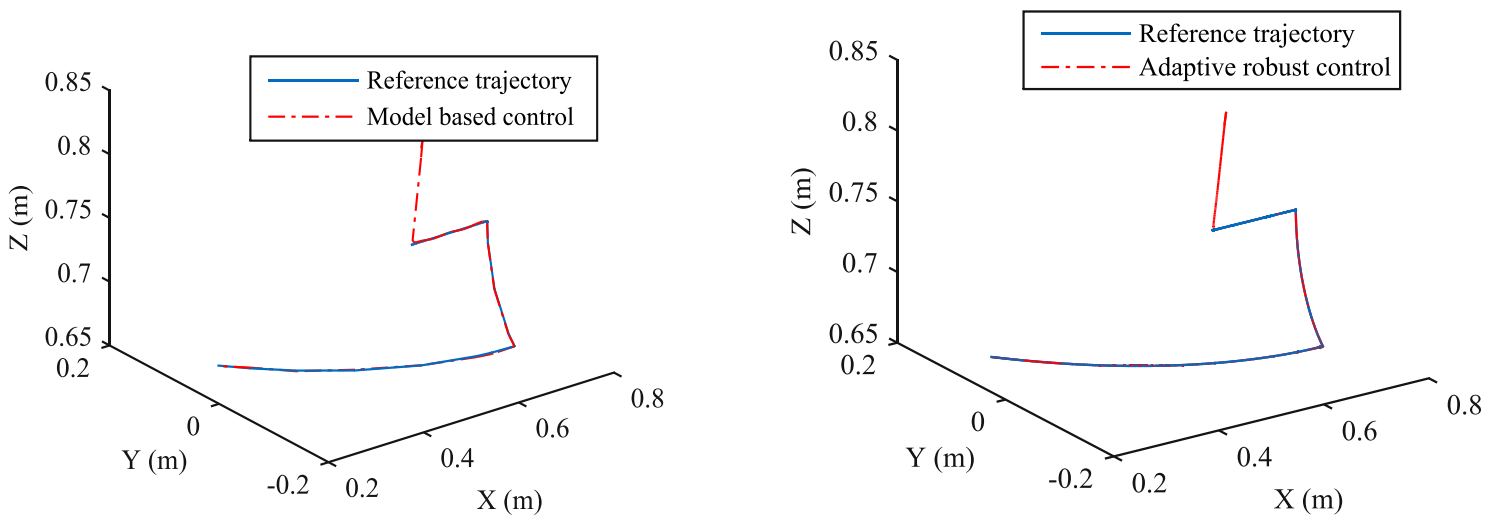

Figure 5: The end-effector trajectory tracking under model-based control and robust adaptive control.
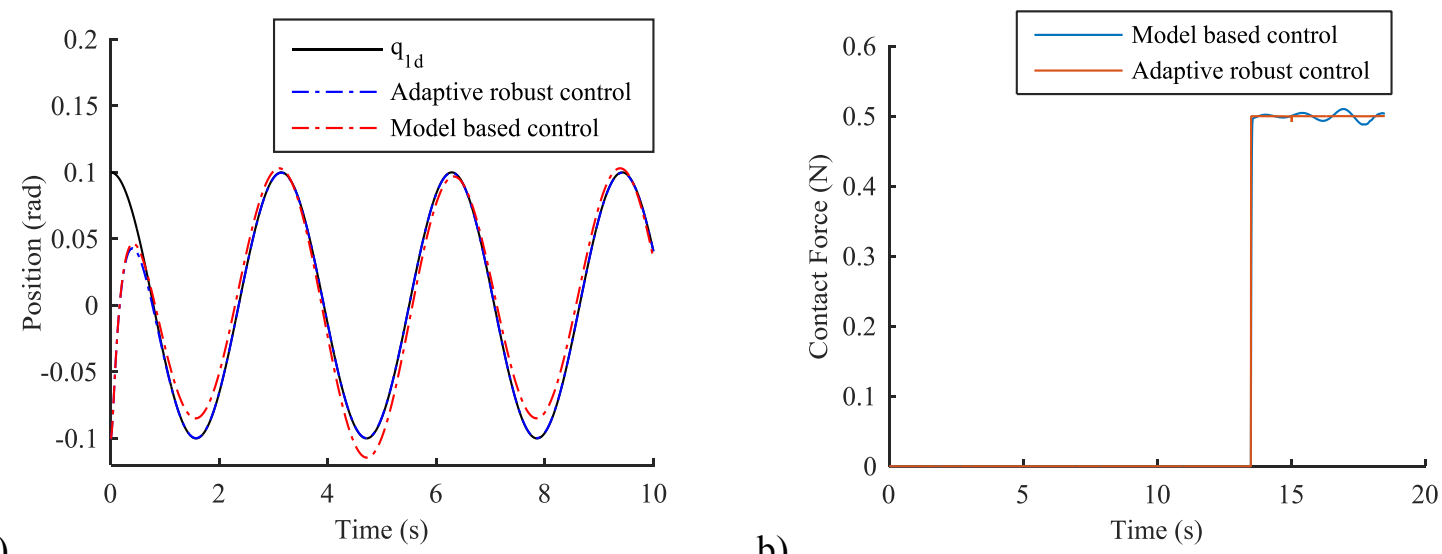

a)

b)

Figure 6: a) Position tracking of the waist joint under model-based control and robust adaptive control, b) Constraint force tracking under model-based control and robust adaptive control.

The simulations are conducted by the model-based control and the adaptive robust control under the same initial conditions and the same operation environment. The system model in simulations has $10 \%$ uncertainty. The trajectory tracking performances of the model-based control and the adaptive robust control are illustrated in Figs. 4 to 7. Fig. 4 shows the trajectory tracking performances of the vehicle under two control methods. The robust 
adaptive control has smaller tracking errors relative to the model-based control. Figs. 5 and 6 a show the position tracking effects in the operating space of the manipulator and the joint space of the unconstrained waist joint under two different control methods. Fig. $6 \mathrm{~b}$ shows that the constraint force has a large fluctuation under the model-based control and a gentle force under robust adaptive control. The robust adaptive control is more effective than the modelbased control. The sensor feedback signals indicate that the reference trajectory is predefined before opening a door. The position deviation can be compensated for according to the force feedback when opening a door. Fig. 7 verifies good tracking effects of the Euler angles under the adaptive robust control. The Euler angles assure that the gripper has the right pose to grasp the door handle. The robust adaptive control can effectively ensure that the rotational velocity of the wrist joint tracks the rotational velocity of the handle; however, the robust adaptive control is smoother, more stable and more rapidly converged.

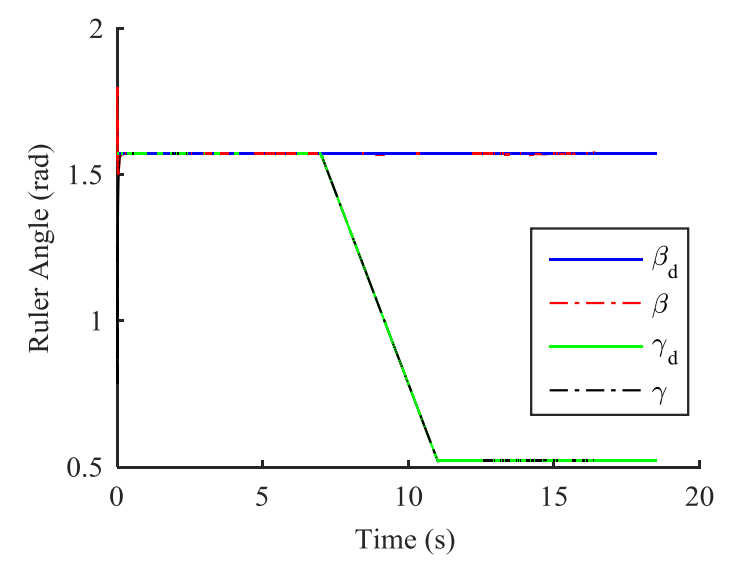

Figure 7: Position tracking of the Euler angles under robust adaptive control.

\subsection{Application to the door-opening system}

The door-opening operation is a typical nonlinear control process with the nonlinear dynamics of the mobile manipulator, which is subjected to holonomic and non-holonomic constraints. Co-simulation experiments between MATLAB and RecurDyn dynamic software are conducted to validate the proposed methods and to verify the complex control in the approximated real scene. The actual door-opening scenario, the simulation scenario and the contact force in opening the door are presented in Fig. 8. The simulation scenario is composed of a door with a handle and a mobile robot with a 5-DOF manipulator. The data of trajectory planning are imported into the RecurDyn dynamic software from the MATLAB software. The door model is designed in RecurDyn according to the actual size of a family door. The entity model of the mobile manipulator is designed in Solidworks and imported into RecurDyn. The constraints and relevant motion functions are imposed on all joints of the door and the mobile manipulator. The door-opening simulations and the adjusted contact force are illustrated in Figs. $8 \mathrm{~b}$ and $8 \mathrm{c}$. The contact force in the experiment is shown in Fig. $8 \mathrm{c}$.

Contact force control is a complex process and varies with the opening angle of the door. The contact force on the gripper is controlled to approximately $16.5 \mathrm{~N}$ in Fig. $8 \mathrm{c}$ through the co-simulation between the MATLAB/Simulink and the RecurDyn. This force is equal to the reaction force from the torsional spring of the door handle, and the friction force caused by this force ensures that the door can be opened. The periods of $0 \mathrm{~s}$ to $8 \mathrm{~s}$ and $8 \mathrm{~s}$ to $25 \mathrm{~s}$ are used in opening the door to approach and rotate the door handle without clenching the handle. The gripper clenches the handle when pulling the door open in the period of $26 \mathrm{~s}$ to $40 \mathrm{~s}$. The clenching operation in the period of $25 \mathrm{~s}$ to $26 \mathrm{~s}$ causes the change in contact force and produces large oscillations. The poor posture of the end-effector results in large contact force 
errors in the period of $36 \mathrm{~s}$ to $40 \mathrm{~s}$, because of less DOFs and the unreasonable position of the mobile manipulator. Thus, the opening angle of the door is only limited to a certain range. The radial contact force in Fig. $8 \mathrm{c}$ is approximately equal to the force acting on the handle by the torsional spring, and almost no other additional forces exist. The practical contact force of about $15 \mathrm{~N}$ in Fig. $8 \mathrm{~d}$ is measured by a force sensitive resistor with a $1000 \mathrm{~Hz}$ sample frequency. The practical measurement and theoretical analysis indicate that the contact force conforms to the expected change rule. Due to modelling errors and a lower sensor precision, there are some differences between the simulations and the experiments. The simulation parameters can be used to guide future actual operations of the mobile manipulator. The experiments further verify the effectivity of the simulation models and the control method.

a)

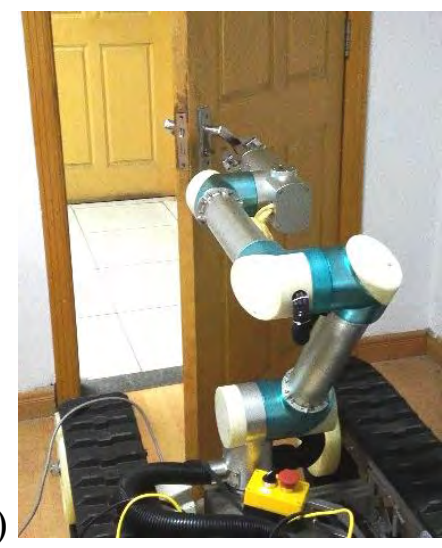

c)

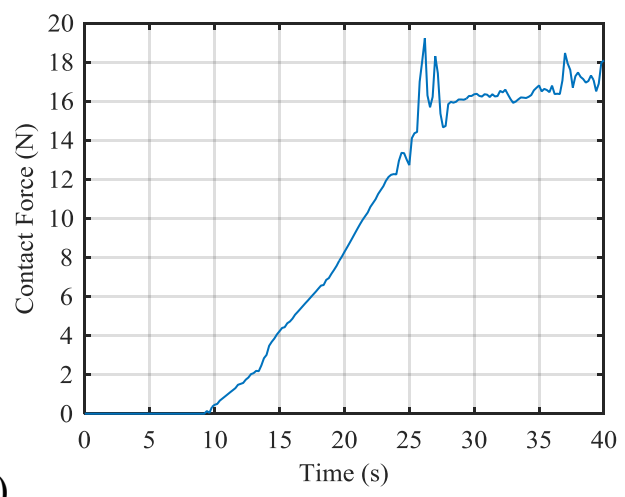

Figure 8: a) Real-world door-opening indoor environment, b) simulation of pulling the door handle, c) contact force in simulation, d) contact force in the experiment.

b)
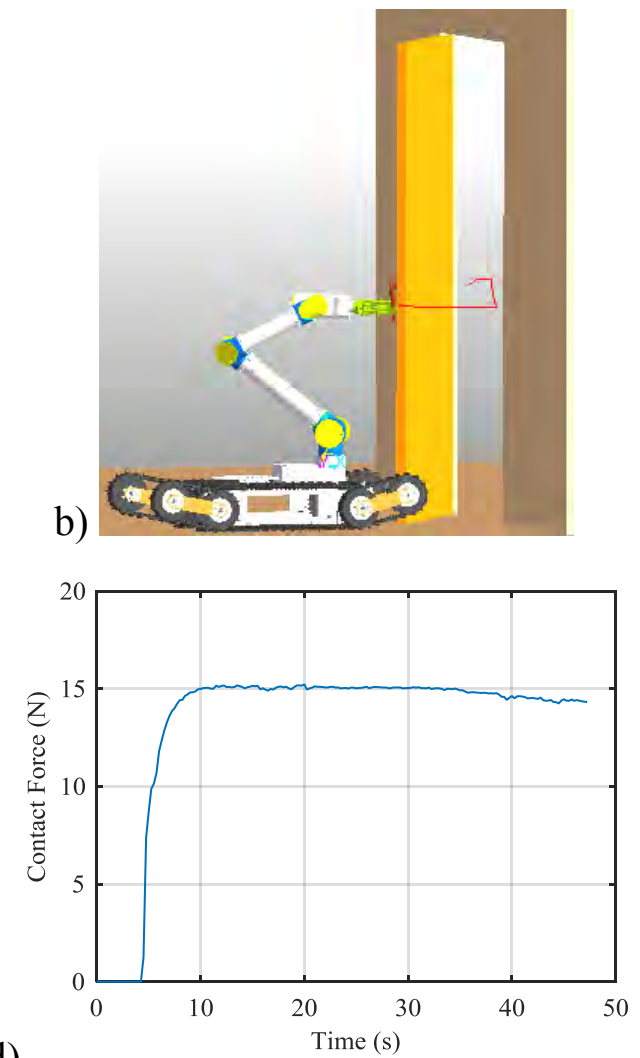

d)

\section{CONCLUSION}

The dynamic simulations of a mobile manipulator were performed in MATLAB and RecurDyn to successfully open a door in a narrow space with a mobile manipulator and suppress the excessive internal force between the gripper and the door handle, respectively. The following conclusions were obtained:

1) The door-opening strategy based on robust adaptive position/force control theory can effectively suppress modelling errors, joint frictions and external disturbances in opening a door and make position and contact force tracking smooth, stable and rapidly converged.

2) Relevant simulation studies and experiments verified that the control model of the dooropening system can effectively perform the door-opening task in a narrow environment and protect the door handle and the gripper against damage from excessive internal forces.

This study can offer reference data on opening a door with a handle to avoid the blindness of parameter settings. However, the preliminary experiments are only made in this study. To 
further validate the proposed method, further studies are needed to solve the controllability and the practicability of the mobile manipulator for different types of doors in different environments.

\section{ACKNOWLEDGEMENT}

This work was supported by the Key Projects of the National Natural Science Foundation of China (61233010), Shanghai Municipal Science and Technology Commission Project (14DZ1110900) and the Outstanding Academic Leaders' Plan of Shanghai (15XD1501800).

\section{REFERENCES}

[1] Zhang, H.-W.; Liu, Y.-G.; Liu, G.-J. (2013). Multiple mode control of a compact wrist with application to door opening, Mechatronics, Vol. 23, No. 1, 10-20, doi:10.1016/ j.mechatronics.2012.10.005

[2] Liu, G.-J.; Liu, Y.-G.; Goldenberg, A. A. (2011). Design, analysis, and control of a springassisted modular and reconfigurable robot, IEEE/ASME Transactions on Mechatronics, Vol. 16, No. 4, 695-706 doi:10.1109/TMECH.2010.2050895

[3] Kessens, C. C.; Rice, J. B.; Smith, D. C.; Biggs, S. J.; Garcia, R. (2010). Utilizing compliance to manipulate doors with unmodeled constraints, Proceedings of the 2010 IEEE/RSJ International Conference on Intelligent Robots and Systems, 483-489, doi:10.1109/IROS.2010.5650927

[4] Meeussen, W.; Wise, M.; Glaser, S.; Chitta, S.; McGann, C.; Mihelich, P.; Marder-Eppstein, E.; Muja, M.; Eruhimov, V.; Foote, T.; Hsu, J.; Rusu, R. B.; Marthi, B.; Bradski, G.; Konolige, K.; Gerkey, B.; Berger, E. (2010). Autonomous door opening and plugging in with a personal robot. Proceedings of the IEEE International Conference on Robotics and Automation, 729-736, doi:10.1109/ROBOT.2010.5509556

[5] Prats, M.; Sanz, P. J.; del Pobil, A. P. (2010). Reliable non-prehensile door opening through the combination of vision, tactile and force feedback, Autonomous Robots, Vol. 29, No. 2, 201-218 doi:10.1007/s10514-010-9192-1

[6] Winiarski, T.; Banachowicz, K.; Stefańczyk, M. (2013). Safe strategy of door opening with impendance controlled manipulator, Journal of Automation, Mobile Robotics \& Intelligent Systems, Vol. 7, No. 4, 21-26, doi:10.14313/JAMRIS 4-2013/21

[7] Zucker, M.; Jun, Y.; Killen, B.; Kim, T.-G.; Oh, P. (2013). Continuous trajectory optimization for autonomous humanoid door opening, Proceedings of the 2013 IEEE International Conference on Technologies for Practical Robot Applications, 5 pages, doi:10.1109/TePRA.2013.6556358

[8] Guo, W.; Wang, J.-C; Chen, W.-D. (2014). A manipulability improving scheme for opening unknown doors with mobile manipulator, Proceedings of the 2014 IEEE International Conference on Robotics and Biomimetics, 1362-1367, doi:10.1109/ROBIO.2014.7090523

[9] Kim, P. K.; Lee, Y.; Park, J. (2011). Twisting door handle with manipulator under uncertainty, Proceedings of the $20118^{\text {th }}$ International Conference on Ubiquitous Robots and Ambient Intelligence (URAI), 525-529, doi:10.1109/URAI.2011.6145876

[10] Chung, W.; Rhee, C.; Shim, Y.; Lee, H.; Park, S. (2009). Door-opening control of a service robot using the multifingered robot hand, IEEE Transactions on Industrial Electronics, Vol. 56, No. 10, 3975-3984 doi: 10.1109/TIE.2009.2025296

[11] Kim, D. W.; Kang, J.-H.; Park, G.-T. (2010). Door-opening behaviour by home service robot in a house, International Journal of Robotics \& Automation, Vol. 25, No. 4, 271 doi:10.2316/ Journal.206.2010.4.206-3195

[12] Lee, J.; Ajoudani, A.; Hoffman, E. M.; Rocchi, A.; Settimi, A.; Ferrati, M.; Bicchi, A.; Tsagarakis, N. G.; Caldwell, D. G. (2014). Upper-body impedance control with variable stiffness for a door opening task, Proceedings of the $201414^{\text {th }}$ IEEE-RAS International Conference on Humanoid Robots (Humanoids), 713-719 doi:10.1109/HUMANOIDS.2014.7041441

[13] Karayiannidis, Y.; Smith, C.; Vina, F. E.; Ogren, P.; Kragic, D. (2012). “Open sesame!” adaptive force/velocity control for opening unknown doors, Proceedings of the 2012 IEEE/RSJ International Conference on Intelligent Robots and Systems (IROS), 4040-4047, doi:10.1109/IROS.2012.6385835 
[14] Schmid, A. J.; Gorges, N.; Goger, D.; Worn, H. (2008). Opening a door with a humanoid robot using multi-sensory tactile feedback, Proceedings of the 2008 IEEE International Conference on Robotics and Automation, 285-291, doi:10.1109/ROBOT.2008.4543222

[15] Winiarski, T.; Banachowicz, K. (2013). Opening a door with a redundant impedance controlled robot, Proceedings of the $20139^{\text {th }}$ Workshop on Robot Motion and Control (RoMoCo), 221-226, doi:10.1109/RoMoCo.2013.6614612

[16] Mohammadi, A.; Tavakoli, M.; Marquez, H. J.; Hashemzadeh, F. (2013). Nonlinear disturbance observer design for robotic manipulators, Control Engineering Practice, Vol. 21, No. 3, 253-267, doi: $10.1016 /$ j.conengprac.2012.10.008

[17] Zhang, Z.-H.; Hu, C. (2015). Multi-model stability control method of underactuated biped robots based on imbalance degrees, International Journal of Simulation Modelling, Vol. 14, No. 4, 647657, doi:10.2507/IJSIMM14(4)7.318

[18] Li, Z.-J.; Yang, Y.-P.; Li, J.-X. (2010). Adaptive motion/force control of mobile under-actuated manipulators with dynamics uncertainties by dynamic coupling and output feedback, IEEE Transactions on Control Systems Technology, Vol. 18, No. 5, 1068-1079, doi:10.1109/ TCST.2009.2033573

[19] Islam, S.; Liu, X. P. (2011). Robust sliding mode control for robot manipulators, IEEE Transactions on Industrial Electronics, Vol. 58, No. 6, 2444-2453, doi:10.1109/TIE.2010. 2062472

[20] Yao, Z.-Q.; Cheng, S.-J.; Pan, T.-H. (2015). An improved model-free adaptive control algorithm for VSC-HVDC power transmission systems, Dyna, Vol. 90, No. 6, 653-661, doi:10.6036/7741

[21] Chen, C.-C; Li, J.-S.; Luo, J.; Xie, S.-R.; Pu, H.-Y.; Cui, Z.; Gu, J. (2014). Analysis and simulation of kinematics of 5-DOF nuclear power station robot manipulator, Proceedings of the 2014 IEEE International Conference on Robotics and Biomimetics (ROBIO), 2025-2030, doi:10.1109/ROBIO.2014.7090634

[22] Tahriri, F.; Mousavi, M.; Yap, H. J.; Siti Zawiah, M. D.; Taha, Z. (2015). Optimizing the robot arm movement time using virtual reality robotic teaching system, International Journal of Simulation Modelling, Vol. 14, No. 1, 28-38, doi:10.2507/IJSIMM14(1)3.273 